\title{
Semigroups with the Erdős-Turán Property
}

\author{
J. Nešetřil \\ Department of Applied Mathematics \\ Institute of Theoretical Computer Science (ITI) \\ Charles University, Praha \\ nesetril@kam.ms.mff.cuni.cz \\ and \\ O. Serra* \\ Dept. Applied Mathematics IV \\ Polytechnical University of Catalonia, Barcelona \\ oserra@ma4.upc.es
}

\begin{abstract}
A set $X$ in a semigroup $G$ has the Erdős-Turán property $E T$ if, for any basis $A$ of $X$, the representation function $r_{A}$ is ubounded, where $r_{A}(x)$ counts the number of representations of $x$ as a product two elements in $A$. We show that, under some conditions, operations on binary vectors whose value at each coordinate depends only on neighbouring coordinates of the factors give rise to semigroups with the ET-property. In particular countable powers of semigroups with no mutually inverse elements have the $E T$-property. As a consequence, for each $k$ there is $N(k)$ such that, for every finite subset $X$ of a group $G$ with $X \cap X^{-1}=\{1\}$, the representation function of every basis of $X^{N} \subset G^{N}, N \geq N(k)$, is not bounded by $k$. This is in contrast with the known fact that each $p$-elementary group admits a basis of the whole group whose representation function is bounded by an absolute constant.
\end{abstract}

\section{Introduction}

Let $(G, *)$ be a set with a binary operation and $X \subset G$. A subset $A \subset X$ is a basis of $X$ if $X \subset A * A$. When $X$ is an infinite set, $A$ is an asymptotic

*Supported by the Spanish Research Council under project BFM2002-00412 
basis if $X \backslash(A * A)$ is finite.

For $g \in G$ we denote by $r_{A}(g)$ the number of pairs $\left(a, a^{\prime}\right) \in A \times A$ such that $g=a * a^{\prime}$. The function $r_{A}$ is the representation function of $A$.

Given a positive integer $k$, we say that $X$ has the $k$-Erdös-Turán property $E T(k)$ if, for any base $A$ of $X$, there is an element $x \in X$ with $r_{A}(x) \geq k$. We say that $X$ has the Erdös-Turán property ET if it has $E T(k)$ for every $k \in \mathbb{N}$.

A famous conjecture of Erdős and Turán [5] formulated in 1942 states that the set of positive integers with addition has the ET property. The conjecture was proved to be true for the class of so-called $d$-bounded additive basis of $\mathbb{N}$, see [10]. However it is still wide open in its general formulation. Erdös showed that the function $r_{A}(n)$ can have logarithmic growth. Ruzsa [12] gives a construction of a base $A$ for which the number of representations is bounded in the square mean. These results indicate the difficulties involved in the conjecture and leads to the consideration of the problem in other semigroups.

The situation for the integers with multiplication is different. Erdös [1] proved in 1964 that $(\mathbb{N}, \cdot)$ does have the ET property. Nešetřil and Rödl [9] gave a simple proof of this result by using Ramsey Theorem. Puš [11] extended this result by showing that an abelian semigroup with an infinite set of primes and a finite number of units has the ET property.

On the negative side, it is not difficult to show that the group of integers with addition has basis with unique representation (up to commutativity), a result which can be extended to any abelian free group. Nathanson [8] even showed that the direct product of a countable semigroup with an infinite abelian group $G$ such that $\{12 g ; g \in G\}$ is infinite, admits an asymptotic base whose representation function can be arbitrarily prescribed. Ruzsa [12] shows that, for any prime $p$ such that 2 is a square, there are bases of $\mathbb{Z}_{p} \times \mathbb{Z}_{p}$ whose representation function is bounded by 18 . This result has been recently extended by Haddad and Helou [6] to the additive group of $F \times F$ for any finite field $F$ of odd order. These authors also show [7] that there is an absolute constant $C$ such that no cyclic group has the property $E T(k)$ for $k>C$. In particular, the $p$-elementary groups with $p>2$ do not have the $E T(k)$-property for $k>18 C$.

In this paper we place the Ramsey argument of [9] in a natural broader setting by considering operations in the set of binary vectors. We show that locally bounded operations, a notion explained in Section 2, on binary 
vectors of length $N$ do have the $E T(k)$ property for each positive integer $k$ and large enough $N$. This result allows one to show that several classes of semigroups have the ET property. Among these there are the class of direct products of semigroups with no mutually inverse elements, the class of finite or cofinite sets of a countable set with union, or the class $\left\{\left(\mathbb{N}^{k},+\right), k \in \mathbb{N}\right\}$ of powers of $\mathbb{N}$ with componentwise addition.

As a consequence of these results, combined with the negative results for abelian groups mentioned above, we can formulate the following statement:

Theorem 1 Let $G$ be a group of prime order $p>2$ and $k$ a positive integer. Let $R \subset G$ such that $R \cap(-R)=\{0\}$. There is $N(k)$ such that $R^{N} \subset G^{N}$ has the $E T(k)$ property for each $N \geq N(k)$, while $G^{N}$ has basis $A$ with representation function bounded by $18 C$.

Theorem 1 shows that there are abelian groups which admit basis with bounded representation function, while containing asymmetric subsets with the ET property. The Erdös-Turán conjecture says that this is the case for the group of integers.

\section{Locally bounded operations on binary vectors}

Let $B_{N}=\{0,1\}^{N}$ denote the set of all binary vectors of length $N$. We denote by $s(x)$ the support of vector $x \in B_{N}$, that is, the set of nonzero coordinates of $x$. We denote by $B_{\mathbb{N}}$ the set of infinite binary sequences with finite support.

Given a nonnegative integer $r$ we denote by $I_{r}=[-r, r]$ the integer interval of length $2 r+1$ centered at 0 . The $r$-neighborhood of a subset $U \subset[1, N]$ is defined as the set $(\bar{U})_{r}=\left(\bigcup_{i \in U}\left(i+I_{r}\right)\right) \cap[1, N]$. For a vector $x \in B_{N}$ we denote by $(x)_{U}=\left(x_{i} ; i \in U\right)$ the vector of length $|U|$ of the entries of $x$ in $U$.

Let $\alpha: \mathbb{N}_{0} \rightarrow \mathbb{N}_{0}$ be a function. A binary operation ' $*$ ' defined in $B_{N}$ is said to be $\alpha$-locally bounded if, for every three vectors $x, x^{\prime}, y$, and every $i$, $1 \leq i \leq N$, the following conditions hold:

$$
\begin{array}{r}
\text { if }(x)_{(\bar{i})_{r}}=\left(x^{\prime}\right)_{(\bar{i})_{r}} \text { then also }(x * y)_{i}=\left(x^{\prime} * y\right)_{i}, \\
\text { if }(x)_{(\bar{i})_{r}}=(0, \cdots, 0) \text { then }(x * y)_{i}=(y * x)_{i}, \\
s(x * y) \subset(\overline{s(x) \cup s(y)})_{r} \text { and } s(x) \cup s(y) \subset(\overline{s(x * y)})_{r} .
\end{array}
$$


where $r=\alpha(|s(x * y)|)$. Condition (1) says that the $i$-th coordinate of $x * y$ depends only on the coordinates of $x$ and $y$ in the $r$-neighborhood of $i$. This is the essential property of a locally bounded operation. Condition (3) specifies that the $r$-neighborhood of a coordinate $i$ in $x * y$ is the zero vector only if both $x$ and $y$ have zero entries in the iterated $r$-neighborhood of $i$, while (2) states that the zero vector commutes 'locally' with every vector.

Theorem 2 Let $k$ be a positive integer and $\alpha: \mathbb{N}_{0} \rightarrow \mathbb{N}_{0}$ a function. There is $N(k, \alpha)$ such that $\left(B_{N}, *\right)$ has the $E T(k)$-property for every $\alpha$-locally bounded operation ' $*$ ' on $B_{N}$ and all $N \geq N(k, \alpha)$.

Proof. Given $k$, let $t=\left\lceil\log _{2} k+1\right\rceil$ and set $r=\alpha(t)$. Define $N=N(k, \alpha)=$ $(5 r+1)\left(R\left(2 t^{2}, t, 2^{t(2 r+1)}\right)+2\right)$, where $R(m, t, l)$ denotes the Ramsey number which ensures the existence of an homogeneous subset of $[1, R(m, t, l)]$ of cardinality $m$ all of whose $t$-subsets are monochromatic for any coloring of the $t$-subsets with $l$ colors.

Let $A$ be a basis of $\left(B_{N}, *\right)$.

Take the subset $Y \subset[1, N]$ of multiples of $5 r$ if $r \geq 1$ and $Y=[1, N]$ if $r=0$. Define a coloring $c$ of the $t$-subsets of $Y$ as follows. Given a $t$-subset $X=\left\{i_{1}<i_{2}<\cdots<i_{t}\right\}$, let $x$ be the vector with the support $X$. Choose a pair $y, z \in A$ such that $x=y * z$. By (3) we have $s(x) \subset(\overline{s(y) \cup s(z)})_{r}$. Since two consecutive elements in $s(x)$ are at least at distance $4 r+1$, we can choose one of $y$ and $z$, say $y$, such that $s(y)$ intersects at least $t / 2$ of the disjoint intervals $\left(\overline{i_{1}}\right)_{r},\left(\overline{i_{2}}\right)_{r}, \ldots,\left(\overline{i_{t}}\right)_{r}$. Color $X$ by the binary vector of length $r t$ given by

$$
c(X)=\left((y)_{\left(\overline{i_{1}}\right)_{r}},(y)_{\left(\overline{i_{2}}\right)_{r}}, \ldots,(y)_{\left(\overline{i_{t}}\right)_{r}}\right) .
$$

By (3) again, we have $s(y) \subset(\overline{s(x)})_{r}$, so that this coloring gives an encoding of $y$ except that we do not keep track of the position of its support in $[1, N]$.

Since $A$ is a base, $c$ is a coloring of all $t$-subsets of $Y$ and it uses at most $2^{t(2 r+1)}$ colors. By the definition of $N$, there is a subset $Z \subset Y$ of cardinality $|Z|=2 t^{2}$ all of whose $t$-subsets have the same color $u=\left(u_{1}, u_{2}, \ldots, u_{t r}\right)$.

Let $u^{(1)}=\left(u_{1}, \ldots, u_{r}\right), u^{(2)}=\left(u_{r+1}, \ldots u_{2 r}\right), \ldots, u^{(t)}=\left(u_{(t-1) r+1}, \ldots u_{t r}\right)$. By the construction of the coloring, at least $t / 2$ of the vectors $u^{(1)}, u^{(2)}, \ldots, u^{(t)}$ have nonempty support. Let $u^{\left(i_{1}\right)}, u^{\left(i_{2}\right)}, \ldots, u^{\left(i_{s}\right)}$ be these vectors.

Choose a subset $J=\left\{j_{1}<j_{2}<\cdots<j_{2 s}\right\} \subset Z$ of cardinality $2 s$ such that there are at least $t$ elements of $Z$ between two consecutive elements of $J$. 
Denote by $J_{i}=\left\{j_{2 i-1}, j_{2 i}\right\}, 1 \leq i \leq s$. Recall that, by the choice of $Y$, every two consecutive elements in $J$ are at distance at least $t(4 r+1)$.

Let $K=\left\{k_{1}<\cdots<k_{s}\right\}$ be a $s$-subset of $J$ obtained by picking one element in each $J_{i}$. Since there are $t$ elements of $Z$ between any two consecutive elements in $J$, this set can be completed to a $t$-subset by inserting an element from $Z \backslash J$ in each position $i$ such that $u^{(i)}$ is the zero vector. In other words, we construct a $t$-set $K^{\prime}=\left\{k_{1}^{\prime}, \cdots<k_{t}^{\prime}\right\} \subset Z$ such that $k_{i_{l}}^{\prime}=k_{l}$ for $l=1, \ldots s$.

Since $Z$ is an homogeneous set, the vector $a=a(K) \in B_{N}$ whose support is contained in $(\bar{K})_{r}$ and

$$
(a)_{(\bar{K})_{r}}=\left(u^{\left(i_{1}\right)}, u^{\left(i_{2}\right)}, \ldots, u^{\left(i_{s}\right)}\right)
$$

belongs to the base $A$. By the same argument, the similarly defined vector $a(J \backslash K)$ also belongs to the base. Denote by $b(K)$ the vector which coincides with $a(K)$ on the $r$-neighborhood of $K$ and with $a(J \backslash K)$ in the $r$-neighborhood of $J \backslash K$ and has zero coordinates elsewhere (this is a correct definition as in our situation these $r$-neighborhoods are pairwise disjoint.) We have

$$
(b(K))_{(\bar{J})_{r}}=\left(u^{\left(i_{1}\right)}, u^{\left(i_{1}\right)}, u^{\left(i_{2}\right)}, u^{\left(i_{2}\right)}, \ldots, u^{\left(i_{s}\right)}, u^{\left(i_{s}\right)}\right) .
$$

By (3), the support of $a(K) * a(J \backslash K)$ is contained in the $r$-neighborhood of $s(b(K))=s(a(K)) \cup s(a(J \backslash K))$. This $r$-neighborhood is a subset of $\left(\overline{(\bar{J})_{r}}\right)_{r}$. Since two consecutive elements in $J$ are at least at distance $4 r+1$, the set $(\overline{s(b(K))})_{r}$ is again the disjoint union of the $r$-neighborhoods of each element in $s(b(K))$.

Let $K^{\prime} \subset J$ be another choice of a $s$-subset with exactly one element in each $J_{i}$. The vectors $a(K)$ and $a\left(K^{\prime}\right)$ differ in the $r$-neighborhood of the symmetric difference $K \Delta K^{\prime}$. On the other hand, $b(K)=b\left(K^{\prime}\right)$. By (1) and (2), for each coordinate $i$ in the $r$-neighborhood of $\left(\overline{K^{\prime} \Delta K}\right)_{r}$ we have $\left(\left(a\left(K^{\prime}\right) * a\left(J \backslash K^{\prime}\right)\right)_{i}=\left((a(J \backslash K) * a(K))_{i}=\left(a(K) *(a(J \backslash K))_{i}\right.\right.\right.$, while for the remaining coordinates in $\left.\overline{(\bar{J})_{r}}\right)_{r}$, we have $\left(\left(a\left(K^{\prime}\right) * a\left(J \backslash K^{\prime}\right)\right)_{i}=\right.$ $\left((a(K) * a(J \backslash K))_{i}\right.$. Therefore

$$
a\left(K^{\prime}\right) * a\left(J \backslash K^{\prime}\right)=a(K) * a(J \backslash K) .
$$

There are $2^{s-1}$ choices leading to different pairs of vectors $\{a(K), a(J \backslash K)\}$. Hence, for $u=a(K) * a(J \backslash K)$, we have

$$
r_{A}(u) \geq 2^{t / 2-1} \geq k .
$$

This completes the proof. 


\section{Semigroups with the Erdős-Turán property}

Let $(G, *)$ be a semigroup with a distinguished idempotent element $e \in G$ which commutes with every element in $G$. We say that a subset $R \subset G$ is antisymmetric if $e \in R$ and the equation $x * y=e$ holds for $x, y \in R$ if and only if $x=y=e$. We will consider the direct product $G^{N}$. By the support of an vector $g \in G^{n}$ we mean the set of coordinates of $g$ which are different from $e$. We denote by $G^{\mathbb{N}}$ the set of infinite sequences of elements of $G$ with finite support, where the product is defined componentwise. As a direct application of Theorem 2 we have the following result.

Theorem 3 Let $(G, *)$ be a semigroup and $e \in G$ an idempotent element which commutes with every element in $G$. Let $R$ be a finite antisymmetric set of a semigroup with $|R|>1$. For each positive integer $k$ there is $N(k)$ such that $R^{N}$ has the $E T(k)$ property for all $N \geq N(k)$. In particular, $R^{\mathbb{N}}$ has the ET property.

Proof. $\quad$ Define an encoding $\phi$ of the elements of $R$ by binary vectors of length $m=\left\lceil\log _{2}|R|\right\rceil$ such that $\phi(e)=(0, \cdots, 0)$ and $\phi(x)=(0, \ldots, 0,1)$ for some element $x \neq e$ in $R$. Let $B_{N m}^{\prime}=(\phi(R))^{N} \subset B_{N m}$ and define an operation in $B_{N m}^{\prime}$ according to the operation in $G$, that is, for $x, y \in B_{N m}^{\prime}$,

$(x * y)_{[(i-1) r+1, i r]}=\phi\left(\phi^{-1}(x)_{[(i-1) r+1, i r]} * \phi^{-1}(y)_{[(i-1) r+1, i r]}\right) \quad i=1,2, \ldots, m$.

In this way we have a locally bounded operation in $B_{N m}^{\prime}$ with $\alpha(t)=m t$. Indeed, condition (1) is verified by definition, condition (3) follows from the asymmetry of $R$ and (2) holds since $e$ commutes with every element in $G$. We can now apply the proof of Theorem 2 even if the operation is not defined for all vectors in $B_{N m}$ : all vectors whose support lies in the set $Y \subset[1, N m]$ of coordinates multiple of $5 r$ in that proof do belong to $\phi(\{e, x\})^{N} \subset B_{N m}^{\prime}$, and we only use the fact that each of these vectors belong to $A * A$ for any base $A$, which is the case in our present situation. This shows that $G^{N}$ has the $E T(k)$ property for $N \geq(5 m t+1)\left(R\left(2 t^{2}, t, 2^{t(2 m t+1)}\right)+2\right)$ where $t=\left\lceil\log _{2} k+1\right\rceil$.

The result follows for $R^{\mathbb{N}}$ since each of its basis contains a base of $R^{N}$ for every $N$.

The positive part of Theorem 1 follows from Theorem 3 by taking an antisymmetric set in $\mathbb{Z} / p \mathbb{Z}$. 
Let $\mathcal{G}$ be a class of semigroups and $\mathcal{R}$ a family of subsets of $\cup_{G \in \mathcal{G}} G$. We say that $\mathcal{R}$ has the $E T$-property if for every $k$ there is $N(k)$ such that every member $R \in \mathcal{R}$ with $|R| \geq N(k)$ has the $E T(k)$-property. As a specialization of Theorem 3 we have the following examples of semigroups with the Erdős-Turán property.

Corollary 1 The following classes have the ET property.

1. $\left\{\left(P^{N}, \vee\right), N \in \mathbb{N}\right\}$ and $\left(P^{\mathbb{N}}, \vee\right)$, where $P$ is a finite semilattice with supremum and with a minimum element.

2. The family of finite (or cofinite) subsets of a countable set $X$ with respect to union, $\left(2^{X}, \cup\right)$, and with respect to intersection, $\left(2^{X}, \cap\right)$.

3. $\left\{\left([0, m]^{N},+\right), N \in \mathbb{N}\right\}$ and $\left([0, m]^{\mathbb{N}},+\right)$, where $[0, m]$ is the interval of integers $0 \leq i \leq m$ and the sum is componentwise.

4. $\left\{\left(\mathbb{N}^{N},+\right), N \in \mathbb{N}\right\}$ and $\left(\mathbb{N}^{\mathbb{N}},+\right)$, where the sum is componentwise.

Proof. For $\left\{\left(P^{N}, \vee\right), N \in \mathbb{N}\right\}$ and for $\left(P^{\mathbb{N}}, \vee\right)$, the conditions of Theorem 3 are satisfied with $R=P$ and $e$ the minimum element of $P$.

In particular, for $P=\{0,1\}$ with the usual supremum function, $\left(P^{k}, \vee\right)$ corresponds to the family of subsets of $[1, N]$ with union and $\left(P^{\mathbb{N}}, \vee\right)$ to the family of finite subsets of an infinite countable set. By taking complements we get the result for cofinite sets with intersection. To prove (2) it remains to show that the class of finite subsets of $\mathbb{N}$ with intersection does have the $E T$-property. Let $A$ be a basis of $\left(2^{\mathbb{N}}, \cap\right)$ and $X$ a nonempty set in $A$. Since for each integer $n$ the set $X \cup\{n\}$ must be obtained as the intersection of two sets in $A$, there are infinitely many sets in $A$ containing $X$ and, for each such set $Y, X$ itself can be written as $X \cap Y$.

Part (3) follows directly from Theorem 3 with $R=[0, m]$. Finally, (4) follows from (3) since every base of $\left(\mathbb{N}^{k},+\right)$ contains a base of $[0, m]^{k}$ for each $m \geq 1$.

Note that the multiplicative semigroup of the positive integers can be viewed as $\left(\mathbb{N}^{\mathbb{N}},+\right)$ by considering a vector $\left(x_{i}, i \in \mathbb{N}\right)$ with finite support as the integer $\prod_{i \in N} p_{i}^{x_{i}}$ where $p_{1}, p_{2}, \ldots$ is the sequence of prime numbers. Thus Corollary 1 (4) includes the result that $(\mathbb{N}, \cdot)$ has the ET property. 


\section{Final remarks}

The condition in Theorem 3 that the set $R$ is antisymmetric is essential for the proof. Although we were always more interested in the positive results (i.e. structures with the $E T$ property) we add a few examples in the opposite direction. We say that a basis $A$ of an abelian semigroup $(G, *)$ is a unique representation basis if every element $g \in G$ can be uniquely represented in $A * A$ (up to commutativity.) A simple greedy algorithm produces unique representation basis of $G^{\mathbb{N}}$ for certain semigroups with units.

Proposition 1 Let $(G, *)$ be an abelian semigroup with identity e and $a, b \in$ $G \backslash\{e\}$ two distinct elements such that $a * b=e$ and $a^{2}, b^{2}$ and 0 are pairwise distinct. Let $R \subset G$ containing $a, b$ and e. Then $R^{\mathbb{N}}$ has unique representation basis.

Proof. Take a linear ordering in $R$ and consider the following ordering in $R^{\mathbb{N}}: x \leq y$ if and only if either $\max s(x)<\max s(y)$ or $\max s(x)=$ $\max s(y)=m$ and $x$ is smaller than $y$ in lexicographic order.

Construct recursively a basis $A$ as follows. Let $A_{0}=\{e\}$. For each $i>0$, let $g$ be the minimum element which is not in $A_{i} * A_{i}$. Let $m_{i}$ the largest element in the support of elements in $\left(A_{i} * A_{i}\right) \cup\{g\}$. Let $g_{a}$ coincide with $g$ except that the $\left(m_{i}+1\right)$-th coordinate of $g_{a}$ is $a$. Let all the coordinates of $g_{b}$ be $e$ except $\left(g_{b}\right)_{m_{i}+1}=b$. Now define $A_{i+1}=A_{i} \cup\left\{g_{a}, g_{b}\right\}$.

We have $g \in A_{i+1} * A_{i+1}$. Moreover, if every element in $A_{i} * A_{i}$ can be uniquely expressed, then the same is true in $A_{i+1} * A_{i+1}$ since each product involving any of the two new elements takes a different value on the $\left(m_{i}+1\right)$ th coordinate. Therefore, $A=\cup_{i \in \mathbb{N}} A_{i}$ is a unique representation basis.

A similar argument as in the above proof shows that finitely generated free groups do not have the ET-property.

Proposition 2 Let $X$ be a finite set. The free group $F(X)$ generated by $X$ does not have the ET property.

Proof. Consider the Cayley graph $\Gamma$ of $F(X)$ with respect to $X$. Order the elements of $F(X)$ by its distance to the identity $e$ in $\Gamma$ and lexicographically within a sphere of radius $k$ with respect to some ordering of the elements in $X$. 
As in Proposition 1, construct recursively a basis $A$. Let $A_{0}=\{e\}$. For each $i>0$, let $g$ be the minimum element which is not in $A_{i} * A_{i}$. Let $k$ be the largest distance to $e$ in $\Gamma\left(A_{i} * A_{i}\right) \cup\{g\}$. Let $g_{1}=g x^{2 k+1}$ and $g_{2}=x^{-2 k-1}$. Now define $A_{i+1}=A_{i} \cup\left\{g_{1}, g_{2}\right\}$. We have $g=g_{1} g_{2} \in A_{i+1} * A_{i+1}$ and any product involving any of the two new elements is at distance at least $k+1$ from $e$ in $\Gamma$. Therefore, $A=\cup_{i \in \mathbb{N}} A_{i}$ is a unique representation basis.

On the other hand, the problem for free semigroups is as hard as for additive basis of positive integers.

Proposition 3 Let $X$ be a finite set. The free semigroup $F S(X)$ generated by $X$ has the ET-property if and only if $(\mathbb{N},+)$ has the ET-property.

Proof. For the if part, note that any base $A$ of $F S(X)$ contains a base of the semigroup generated by a single element, which is isomorphic to $(\mathbb{N},+)$.

Suppose now that $F S(X)$ has the $E T$-property. For every base $A \subset \mathbb{N}$ consider the set $A^{\prime}$ of words in $\mathbb{N}$ whose lengths belong to $A, A^{\prime}=\{w \in$ $F(X):|w| \in A\}$. This is clearly a base of $F S(X)$. If $w=x_{1} * y_{1}=\cdots=$ $x_{k} * y_{k}$ are $k$ different representations of a word $w \in F S(X)$ in elements of the basis $A^{\prime}$, then $\left|x_{1}\right|+\left|y_{1}\right|=\cdots=\left|x_{k}\right|+\left|y_{k}\right|$ are $k$ different representations of $|w|$ in elements of $A$. This shows the only if part.

We have dealt with basis of order two. More generally, for an integer $h \geq 2$, a subset $A \subset X$ is a basis of $X$ of order $h$ if $X \subset \underbrace{A * A * \cdots * A}_{h}=A^{h}$.

Accordingly we say that $X$ has the $E T_{h}(k)$ property if, for every base of order $h$, there is an element in $X$ with at least $k$ representations in $A^{h}$. If $X$ has $E T_{h}(k)$ for every $k \in \mathbb{N}$ then we say that it has the $E T_{h}$ property. The proof of Theorem 2 can be easily extended to prove the following statement.

Theorem 4 Let $h \geq 2$ and $k$ be positive integers and $\alpha: N_{0} \rightarrow \mathbb{N}_{0}$ an arbitrary function. There is $N(h, k, \alpha)$ such that $\left(B_{N}, *\right)$ has the $E T_{h}(k)-$ property for every $\alpha$-locally bounded operation ' $*$ ' on $B_{N}$ and all $N \geq$ $N(h, k, \alpha)$. In particular, $R^{N}$ has the $E T_{h}(k)$-property for every antisymmetric set $R$ with respect to $e$ in a semigroup $G$, where $e$ is an idempotent element commuting with all elements in $R$, and all $N$ large enough.

In connection with the Erdős-Newman problem (cf. [3]) we may ask the following question. Let $A$ be a basis with unbounded representation function 
and let $A=A_{1} \cup \cdots \cup A_{r}$ be a finite partition of $A$. Is it true that one of the parts still has unbounded representation function? The answer is negative in general. It is shown in [10] that there are bases of $\mathbb{Z}$ with unbounded representation function which can be split into two $B_{2}^{1}$ sequences (every element can be uniquely written in each of the parts). It is perhaps true that the question has a positive answer for semigroups with the ET property. Again, the proof of Theorem 2 can be adapted to prove the following.

Theorem 5 Let '*' be a locally bounded operation on $B_{\mathbb{N}}$. Let $A$ be a basis of $B_{\mathbb{N}}$. For every finite partition of $A$ one of the parts has unbounded representation function.

\section{References}

[1] P. Erdős, On the multiplicative representation of integers, Israel J. Math. 2 (1964), 251-261.

[2] P. Erdős, Problems and results in additive number theory, Colloque sur la Theorie des Nombres (CBRM), Bruxelles, 1956, 127-137.

[3] P. Erdős, R.L. Graham, Old and New Problems and Results in Combinatorial Number Theory, LEnseignement Math. 28 (1980), 128 pp.

[4] P. Erdős, Some applications of Ramsey's theorem to additive number theory, European. J. Combin. 1 (1980) 43-46.

[5] P. Erdős and P. Turán, On a problem of Sidon in additive number theory and some related problems, J. London Math. Soc. 16 (1941), 212-215.

[6] L. Haddad, C. Helou, Bases in some additive groups and the Erdős-Turán conjecture, J. Combin. Th. A 108 (2004) 147-153.

[7] L. Haddad, C. Helou, Bases in modular and additive groups, preprint (2004).

[8] M. Nathanson, Representation functions of additive bases for abelian semigroups. Int. J. Math. Math. Sci. 2004, 29-32, (2004), 1589-1597.

[9] J. Nešetřil and V. Rödl, Two proofs in Combinatorial Number Theory, Proc. Am. Math. Soc. 931 (1985), 185-188. 
[10] J. Nešetřil and O. Serra, The Erdős-Turán property for a class of bases, Acta Arith. 115, No.3, (2004), 245-254.

[11] V. Puš, On Multiplicative Bases in commutative semigroups, Europ. J. Combin 3 (1993) 201-211.

[12] I. Ruzsa, A just basis, Mh. Math. 109, (1990) 145-151. 